$\mathrm{KN}-11$

\section{Vagando entre los picos - ¿cómo llegué hasta aquí? Improving methods in data collection and processing}

\author{
Harry Powell ${ }^{1}$ \\ 1. Harry Powell Crystallographic, London, United Kingdom \\ email: hrp1000@virginmedia.com
}

Both data collection and data processing methods have changed dramatically during the history of X-ray crystallography, but the pioneers in the field would still recognise much of what we do today. Developments in electronics (which barely existed when von Laue and the Braggs made their first steps towards structure solution, e.g. [1]) have given rise to huge advances in detector hardware and computational methods, but we still measure the position and intensities of diffraction maxima and use this information to determine structural properties of materials - albeit at a rate that might astonish our forebears.

In a discussion of various moving-crystal and moving-detector methods of data collection, I will discuss how modern data processing has allowed single crystal data collection methods to converge on a moving-crystal-static-detector method that was formalised in the late 1970s [2], just as I left school. I will discuss some of the important milestones together with a view of some of the more interesting side-roads that have been explored over the decades. As I wander amongst the (diffraction) peaks, I will try to answer the question "how did we get here?".

References:

[1] Bragg, W.H. \& Bragg, W.L. (1913) Proc. R. Soc. London Ser. A, 89277 - 291

[2] The Rotation Method in Crystallography (1977), edited by U.W. Arndt \& A.J. Wonacott, North-Holland.K

Keywords: data collection, data processing, historical
$\mathrm{KN}-12$

\section{Free-standing nanostructures at atomic scale: from growth mechanisms to local properties}

Jordi Arbiol $^{1}$

1. Advacned Electron Nanoscopy, ICREA and Catalan Institute of Nanoscience and Nanotechnology (ICN2), CSIC and BIST, Bellaterra, Barcelona, Spain

email: arbiol@icrea.cat

Technology at the nanoscale has become one of the main challenges in science as new physical effects appear and can be modulated at will. Semiconductor materials for electronics, optoelectronics, sensing and energy applications are taking advantage of the low dimensionality, improving their properties and opening a new range of applications. As developments in materials science are pushing to the size limits of physics and chemistry, there is a critical need for understanding the origin of these unique physical properties (optical and electronic) and relate them to the changes originated at the atomic scale, e.g.: linked to structural changes of the material, many times related to the presence of crystal defects.

In the present work, I will show how combining advanced electron microscopy imaging with electron spectroscopy, in an aberration corrected STEM will allow us to probe the elemental composition and electronic structure simultaneously in unprecedented spatial detail.

The talk will focus on several examples in advanced semiconductor nanomaterials for optical, electronic and energy applications. In this way the latest results obtained by my group on direct visualizing and modeling materials at atomic scale will help to understand their growth mechanisms (e.g.: how the occurrence of a single twin defect can modify the growth from 1D (nanowire) to 2D (nanosheet) [1,2]) and also correlate their physical properties (electronic and photonic) at sub-nanometer with their atomic scale crystal structure. The examples will cover a wide range of semiconductor nanomaterials: quantum structures self-assembled in a nanowire: quantum wires (1D) [3] and quantum dots (0D) $[4,5]$ and other complex nanowire-like morphologies for photonic and energy applications (LEDs, lasers, quantum computing, single photon emitters, water splitting cells, batteries) $[7,8,9,10]$, as well as nanomembranes and 2D sheets $[8,11]$.

References:

[1]. de la Mata M, Arbiol J, et al (2014) Nano Letters 14, 6614.

[2]. de la Mata M, Arbiol J, et al (2016) Nano Letters 16, 825.

[3]. Arbiol J et al (2012) Nanoscale 4, 7517.

[4]. Uccelli E, Arbiol J et al (2010) ACS Nano 4, 5985.

[5]. Heiss M, Arbiol J et al (2013) Nature Mater. 12, 439.

[6]. Arbiol J et al (2013) Materials Today 16, 213.

[7]. de la Mata M, Arbiol J et al (2013) J. Mat. Chem. C 1, 4300.

[8]. Tang P-Y, Arbiol J et al (2016) Nano Energy 22, 189.

[9]. Tang P-Y, Arbiol J et al (2017) Energy Environ. Sci., 10, 2124.

[10]. Urbain F, Arbiol J et al (2017) Energy Environ. Sci., 10, 2256.

[11]. Tutuncuoglu G, Arbiol J, et al (2015) Nanoscale 7, 19453.

Keywords: Transmission electron microscopy, nanomaterials, structure 